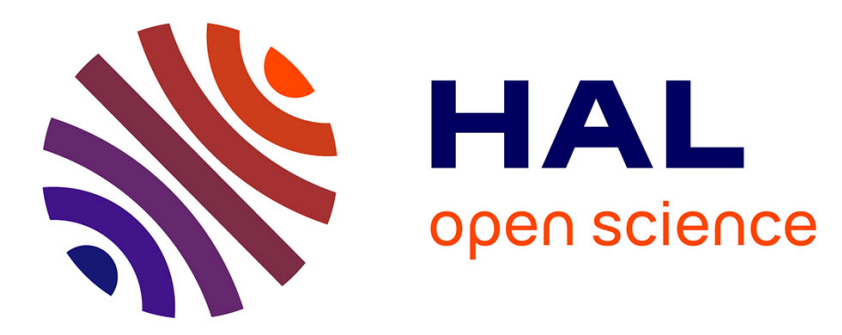

\title{
Targeting TOR signaling for enhanced lipid productivity in algae
}

\author{
Laura Prioretti, Frédéric Carriere, Ben Field, Luisana Avilan, Marie-Hélène
}

Montané, Benoît Menand, Brigitte Gontero

\section{- To cite this version:}

Laura Prioretti, Frédéric Carriere, Ben Field, Luisana Avilan, Marie-Hélène Montané, et al.. Targeting TOR signaling for enhanced lipid productivity in algae. Biochimie, 2020, 169, pp.12-17. 10.1016/j.biochi.2019.06.016 . hal-02176524

\section{HAL Id: hal-02176524 \\ https://hal-amu.archives-ouvertes.fr/hal-02176524}

Submitted on 4 Feb 2020

HAL is a multi-disciplinary open access archive for the deposit and dissemination of scientific research documents, whether they are published or not. The documents may come from teaching and research institutions in France or abroad, or from public or private research centers.
L'archive ouverte pluridisciplinaire HAL, est destinée au dépôt et à la diffusion de documents scientifiques de niveau recherche, publiés ou non, émanant des établissements d'enseignement et de recherche français ou étrangers, des laboratoires publics ou privés. 


\section{Targeting TOR signaling for enhanced lipid productivity in algae}

Laura Prioretti ${ }^{1}$, Frédéric Carriere ${ }^{1}$, Ben Field ${ }^{2}$, Luisana Avilan ${ }^{1}$, Marie-Hélène Montané $^{2}$, Benoît Menand ${ }^{2 *}$, Brigitte Gontero ${ }^{1 *}$

${ }^{1}$ Aix Marseille Univ, CNRS, BIP UMR 7281, 31 Chemin Joseph Aiguier, 13402 Marseille Cedex 09, France

${ }^{2}$ Aix Marseille Univ, CEA, CNRS, UMR 7265 BIAM, 163 Avenue de Luminy, 13288 Marseille, France

*Corresponding authors: bmeunier@imm.cnrs.fr; benoit.menand@univ-amu.fr

\section{Abstract}

Microalgae can be used for many applications, including the development of third generation biofuels. However, the trade-off between biomass production and lipid accumulation and productivity still impairs the viable production of sustainable biofuel from microalgae because neutral lipids accumulate under stress conditions. Recently, in the model marine diatom Phaeodactylum tricornutum, it was shown that inhibiting the target of rapamycin (TOR) kinase using specific ATP-competitive inhibitors could overcome this issue. We believe that basic knowledge in this rather new field is required to develop innovative strategies to improve neutral lipid accumulation in oleaginous microalgae. In this minireview, we therefore focused on the current research on the TOR signaling pathway with a focus on its control of lipid homeostasis. Since TOR has been well-studied in animals, we first describe the effectors of TOR in animal lipogenesis, adipogenesis and lipolysis. We then propose a tentative model of TOR regulation of TAG accumulation in algae, a process that has hitherto been overlooked.

\section{Importance of lipids in $3^{\text {rd }}$ generation biofuels from microalgae}

Lipids, together with carbohydrates, proteins and nucleic acids are essential components of living cells. While glycerophospholipids, sphingolipids, glycolipids and sterol lipids are the main components of cell membranes and can also function as signal transducers, triacylglycerols (TAG) constitute a universal form of transient energy storage in the form of fatty acids esterified with glycerol. They are found for instance in the core of lipid droplets in mammalian adipose tissues, in plant seeds, oleaginous yeasts and microalgae. They are the most abundant neutral lipid species in oils of plant origin. Stored TAGs can fuel the cell in times of nutrient limitation. The pathway leading to neutral lipid synthesis is fairly well conserved among eukaryotes: acetyl-CoA derived from carbon-metabolism is the substrate for de novo fatty acid (FA) synthesis, FAs are 
then conjugated to existing lipophilic alcohols through a series of acylation reactions in which diacylglycerol (DAG) is the last intermediate before TAG [1]. A particularity of plant and algae is that the chloroplast is the major site of de novo FA synthesis [2]. Cellular TAGs and other neutral storage lipids such as steryl esters are stored in lipid droplets, and their content can vary considerably depending on cell type and external environmental cues. For example, plants store TAGs in seeds in order to support the germination and vegetative growth of the next generation, while microalgae and yeast accumulate TAGs during starvation.

In the modern world the increasing energy demand from human activities threatens the availability of finite natural resources and the environment due to the release of $\mathrm{CO}_{2}$ and other pollutants. Against this background, neutral lipids represent a promising renewable resource for the biofuel industry, as well as for human and animal nutrition and health. Oleaginous microorganisms, including certain microalgae, yeasts and bacteria, can accumulate up to $80 \%$ of their biomass in the form of TAGs under appropriate conditions $[3,4]$. These organisms therefore represent a major resource for the development of third generation biofuels. Algae are a polyphyletic group of photosynthetic organisms that evolved from multiple endosymbiotic events. The endosymbiosis of a cyanobacterium by a non-photosynthetic eukaryote led to the Archaeplastida group of chloroplast-containing eukaryotes which includes red algae, glaucophytes, green algae and land plants. Further endosymbiosis of green or red algae by other non-photosynthetic eukaryotes led to the evolution of diverse groups of algae including euglenoids and diatoms [5]. Illustrating this diversity, diatoms have retained unique characteristics from different domains of life [6] such as the presence of the urea cycle [7], which is typical of animals, and a sterol biosynthetic pathway that uses intermediates from both plant and fungal sterol biosynthesis [8]. The use of microalgae for lipid stock production has many advantages that have been widely explored and described [9]: a broad range of microalgal strains can be exploited for oil production [4, 10]; they can reach high growth rates ( 1 to 4 divisions per day for the model green alga, Chlamydomonas reinhardtii [11]); and by consuming $\mathrm{CO}_{2}$ they contribute to reducing greenhouse gas emissions. Moreover, because microalgae can use a variety of other inorganic nutrients they can even be exploited for bio-remediation from wastewaters [3]. Since microalgae are able to grow in controlled environments like that of photobioreactors as well as under extreme conditions (high salinity, and across a broad range of temperature and light intensities), they are not subject to seasonal changes and do not necessarily compete with crops for land and water.

Microalgae typically produce large amounts of lipids under stress conditions such as nutrient limitation, and most notably under nitrogen $(\mathrm{N})$ starvation. However, these stresses interrupt cell proliferation and therefore reduce total biomass and limit the overall lipid yield. In the last decade there has been a gold rush for the selection of strains or the creation of engineered microorganisms able to accumulate more lipids. In this regard a path frequently beaten by researchers is to knockout or overexpress genes involved in carbon and lipid metabolism [10]. However, the growth of these 
strains is often compromised and a better knowledge of the regulation of the balance between growth and lipid accumulation is still required. In this context, a new route is represented by the study of the TOR (Target of Rapamycin) kinase pathway [12], which is a central hub in the regulation of various cell cycle processes including the regulation of nutrient and energy homeostasis, lipid synthesis and is conserved in eukaryotes [13]. We will first present evidence for the importance of TOR signaling in the regulation of lipid homeostasis from the wealth of studies in animals, and then we will present how manipulation of TOR signaling can improve TAG productivity in algae. Finally, we will discuss how recent studies in photosynthetic eukaryotes, in parallel with discoveries on TOR signaling in animals, can be used to understand how TOR regulates TAG accumulation in algae and, ultimately, to target TOR signaling for increased lipid productivity.

\section{The principal TOR signaling effectors that control lipid metabolism in animals}

TOR (Target of Rapamycin) is a large (280 kDa) multi-domain kinase belonging to the phosphatidylinositol kinase-related kinase (PIKK) family. TOR is inhibited by rapamycin (Fig. 1) through interaction with the $12 \mathrm{kDa}$ TOR-FRB domain (FKBP12rapamycin-binding domain). Two canonical TOR containing complexes (TORC), TORC1 and TORC2, exist in both mammals and yeast. Recently, different forms of a TORC3 that differ in structure, function and subcellular localization have been discovered in mammals [14-17]. The TORC1 core, which is composed of the TOR kinase, RAPTOR (regulatory associated protein of TOR) and LST8 (lethal with SEC13 protein 8), is found in all eukaryotes [18] and is sensitive to the allosteric inhibitor rapamycin, which binds to the FRB domain of TOR. TORC1 is regulated by nutrients such as glucose, amino acids, as well as by growth factors and is inhibited by stressrelated signals such as starvation and hypoxia. Conserved functions of TORC1 include the activation of protein, lipid and nucleotide synthesis, as well as promoting progression of the cell cycle. At the same time, TORC1 also inhibits stress-responsive genes and catabolic processes like autophagy $[19,20]$. Upstream signals are funneled towards TORC1 via different repressors and activators that regulate TOR intracellular localization and activity. One of the major upstream repressors of TORC1 is the AMPactivated protein kinase (AMPK), a major sensor of cellular energy status via the AMP/ATP and ADP/ATP ratio in mammals [21]. The orthologues of AMPK in yeast (SNF1, sucrose non fermenting 1) and plants (SnRK1, SNF1-related kinase 1) [22, 23] are not responsive to AMP, indicating that there are distinct strategies for energy sensing in different organisms.

As a general rule, active TOR in mammals promotes lipogenesis and adipogenesis and inhibits lipolysis [24]. Lipid synthesis and storage are promoted by 
the SREBP (sterol regulatory element-binding protein) transcription factor, a master regulator of the transcription of lipo- and sterolgenic genes such as fatty acid synthase, acetyl-CoA carboxylase alpha, stearoyl CoA-desaturase, HMG-CoA reductase, and farnesyl diphosphate synthase [13]. SREBP is retained in the membrane of the endoplasmic reticulum where it is unable to activate transcription. In response to sterol depletion SREBP is cleaved and the SREBP DNA-binding domain is imported into the nucleus where it promotes the transcription of lipogenic enzymes [25]. In mammals, TORC1 regulates the activation of SREBP via two main mechanisms: the phosphorylation of (i) the ribosomal S6 kinase 1 (S6K1) to promote lipogenic processes including the processing of SREBP, and (ii) of lipin-1, a phosphatidic acid phosphatase (PAH) that converts phosphatidic acid (PA) into DAG, an important precursor of membrane and neutral lipids. Upon TORC1 inhibition, lipin-1 becomes dephosphorylated and is imported into the nucleus where it inhibits SREBP to downregulate lipid synthesis. Interestingly, TORC stability and activity is itself dependent on PA, which binds the FRB domain of TOR and acts as an indicator of nutrient sufficiency $[26,27]$. Another S6K1 target, the Serine/Arginine (SR)-rich protein kinase 2 (SRPK2), which controls the expression of lipogenic enzymes was recently reported [28]. It mediates the phosphorylation of SR proteins and their interaction with the spliceosomal protein U1-70K, which is responsible for the splicing of lipogenic pre-mRNAs. The phosphorylation of another mTORC1-S6K target, the glutamylprolyl tRNA synthetase (EPRS), induces EPRS release from the amino acyl tRNA multisynthetase complex and its interaction with the fatty acid transport protein 1, promoting its translocation to the plasma membrane and importing fatty acid to the cells [29]. In addition to inducing lipogenesis and controlling cellular lipid homeostasis in different cell types in the brain and liver, mammalian TORC1 also has a positive effect on the differentiation of stem cells into mature adipocytes and in the formation of adipose tissue [30]. TORC1 controls lipid metabolism in white and brown adipose tissues that respectively favor lipid storage or lipid use. The mobilization of the energy stored in the form of TAG, through the action of several lipases [31] and $\beta$-oxidation of FA can generate either ATP or heat through the uncoupling protein 1 (UCP1) that decouples the mitochondrial electrochemical gradient. These processes, which are still under study, illustrate the fundamental role of TOR in regulating lipid and energy metabolism at the cell and organism level.

\section{TOR inhibition can induce a "get fat growth" regime in algae}

Despite the large diversity and the complex evolution of photosynthetic eukaryotes, all plants and algae investigated contain a conserved TORC1 with at least TOR, RAPTOR and LST8 [32]. No other TOR complexes have yet been described in these groups of organisms. TOR is an essential regulator of proliferation in both plants and algae as shown by the embryo-lethal phenotype of tor null mutants in plants, and 
the repression of both plant and algal growth by the allosteric TOR inhibitor rapamycin or by ATP-competitive TOR inhibitors [33-36].

Two independent studies conducted in plants and algae demonstrated the link between TOR repression and neutral lipid accumulation [37, 38]. Repression of tor expression by inducible RNA silencing in Arabidopsis seedlings led to major metabolic changes including the massive accumulation of storage lipids and starch [37]. Likewise, repression of TOR activity by rapamycin treatment led to increased levels in TAG synthesis-related genes, such as glycerol-3-phosphate acyltransferase and acylCoA:diacylglycerol acyltransferase (DGAT), and consequently to TAG accumulation in Cyanidioschyzon merolae (red algae) and Chlamydomonas reinhardtii (green algae) [39]. These studies were pioneering in that they opened the way to targeting TOR signaling for improving plant and algal lipid production. More recent studies conducted on other diverse groups of microalgae, including the diatom Phaeodactylum tricornutum and the euglenoid Euglena gracilis, showed that TOR inhibition results in the accumulation of large lipid droplets rather similar to those that appear in response to $\mathrm{N}$ starvation [34, 39-42] (Fig. 2). TOR inhibition also induces lipid droplet accumulation in budding yeast, suggesting that this phenotype might be a common feature rather than being specific to photosynthetic eukaryotes [40]. Although TAG accumulation seems to be a general response to TOR inhibition in algae and plants, other associated phenotypes are not always similar. For example, while we observed that $P$. tricornutum treatment with the ATP-competitive TOR inhibitor AZD-8055 (Fig. 1) could lead to a stronger decrease in chlorophyll fluorescence than $\mathrm{N}$ starvation, no significant effect of rapamycin on chlorophyll content was observed in $C$. reinhardtii by Roustan and Weckwerth [43]. However, while ATP-competitive TOR inhibitors can efficiently inhibit TOR, rapamycin is less efficient, at least in animals where it only partially inhibits TOR function [44]. This is probably also the case in algae since rapamycin, even at high concentrations, only partially represses algal growth to $40-50 \%$ of that of untreated cells $[34,35,45,46]$. On the other hand, ATP-competitive TOR inhibitors can almost completely stop algal proliferation [18, 34, 41]. More comparative analyses with similar TOR inhibitors and at similar effective doses are therefore required to accurately compare the extent and diversity of TOR functions in different algae.

As discussed above, a major bottleneck in the development of algae for biofuel production is the fact that most conditions that induce TAG accumulation also repress growth, which strongly limits productivity. Obviously, this is also a concern with TOR inhibitors considering the essential and conserved positive function of TOR in the regulation of cell growth and proliferation. However, analysis of the dose-dependent effects of AZD-8055 in the diatom P. tricornutum allowed the identification of a dose that induced a 3 to 4 fold higher lipid productivity than $N$ starvation. This enhanced lipid productivity was due to a moderated impairment of proliferation that allowed cells to grow and divide while accumulating TAG, in what was coined a "get fat-growth regime" [34]. Notably, it is not possible to titrate $\mathrm{N}$ to achieve the same effect, because $\mathrm{N}$ will be rapidly consumed by the cells. These results show that fine tuning of TOR activity can 
improve lipid productivity, and underline the need to fully decipher the TOR signaling axis involved in lipid homeostasis to improve the balance between proliferation and TAG accumulation.

\section{How could the TOR signaling pathway regulate neutral lipid accumulation in algae?}

Recent phosphoproteomics and pharmacogenetics analyses have identified potential members of the TOR pathway in photosynthetic eukaryotes. Although some of these data were obtained in Arabidopsis and have not yet been confirmed in Chlamydomonas or other more distantly related algae, this section presents an overview of the potentially conserved TOR signaling axis involved in lipid homeostasis, and a tentative model of how TOR might regulate TAG accumulation in algae (Fig. 3).

S6Ks are conserved TOR targets found in plants and algae that are known to regulate translation [47, 48]. Phosphoproteomic studies of Chlamydomonas cells treated with rapamycin or AZD-8055 identified TOR-dependent phosphorylation sites in S6K and ribosomal 66 protein $[43,49]$. In mammals, S6K regulates SREBP processing and other lipogenic genes. However, SREBP homologs are not found in plants and algae. Interestingly, one of the two Arabidopsis S6Ks localizes to the nucleus, suggesting its potential involvement in transcriptional regulation [50]. Orthologues of the SREBP repressor and PAH (lipin-1) are also found in plants and algae [51]. Arabidopsis has two PAHs (PAH1 and 2), whose loss of function induces increase in phospholipid content [51]. In algae species, transcriptomics, proteomics or metabolomics did not report PAHs or PA but this might reflect that such signaling proteins and PA are hard to detect in the nutrient conditions devoted to increase cellular lipid contents [52]. In the green microalga Chlorella vulgaris, proteomics studies reported that S6K increased under nutrient repleted conditions [53] and the phosphatidic acid phosphatase PAP was identified [54]. Strikingly, PAH2 is phosphorylated in a TOR dependent manner in Arabidopsis, suggesting a conservation of the link between TOR and PAH in photosynthetic eukaryotes [55]. PAH1 is also phosphorylated by the cyclin-dependent kinases (CDKs) in Arabidopsis suggesting a further link between lipid homeostasis and cell cycle progression [56]. Hence, although a direct link between S6K/PAH and TAG accumulation has not yet been demonstrated in algae, their conservation strongly suggests that they may play a significant role in algal lipid homeostasis (Fig. 3).

Other potential effectors of TOR signaling were directly identified in genetic screens on algae. First, two members of the DYRK (dual-specificity tyrosinephosphorylation regulated kinase) family of protein kinases were isolated in screens for Chlamydomonas insertional mutants defective for oil and starch accumulation [57, 58]. One, named TAR1 (triacylglycerol accumulation regulator 1 ) is the homolog of the 
yeast and Arabidopsis YAK1 (yet another kinase 1), and the other, named DYRKP, belongs to a plant and algal DYRK subfamily. A link between YAK1/DYRK and TOR signaling was revealed recently in plants during a screen for Arabidopsis mutants resistant to the TOR inhibitor AZD-8055. Arabidopsis YAK1 is a negative regulator of growth that is repressed by TOR [59], interacts with TOR, and is phosphorylated in a TOR-dependent manner [55] but it is not yet clear how TOR-YAK/DYRK signaling affects TAG levels in algae. Another screen aimed at identifying TOR signaling effectors in algae led to the identification of the rapamycin-hypersensitive mutant vip1-1 [60]. VIP1 encodes an inositol hexakisphosphate kinase that pyrophosphorylates the inositol phosphate InsP6 to produce the signaling molecules InsP7 and InsP8. The fact that InsP7 and InsP8 levels drop in rapamycin-treated wild-type cells confirmed a link with TOR signaling [60]. Interestingly, the higher levels of TAGs in the vip1-1 mutant led to the conclusion that InsP7 and InsP8 activate growth at the expense of TAG accumulation [60]. In Arabidopsis, AZD-8055 leads to increased phosphorylation of the VIP1 homolog VIP2 suggesting an indirect regulation by TOR [55]. Interestingly, both tar1 and vip1 mutant phenotypes are dependent on the presence of a carbon source. tar1 mutant fails to accumulate TAGs when $\mathrm{N}$ starvation is coupled to acetate supply (photomixotrophy) but accumulates high level of TAGs in photoautotrophic $\mathrm{N}$-deficient conditions [61]. Similarly, vip1 is hypersensitive to TOR inhibitors on acetate supplied media but not in photoautotrophic conditions [60]. This highlights the importance of the TOR signaling pathway in integrating different nutrient signals to regulate growth and reserve accumulation in algae.

Last but not least, autophagy was the first intracellular process connected to TOR signaling to be reported in algae. Rapamycin treatment was shown to induce vacuolization [35] and post-translational modification of the key autophagy-related protein ATG8 in C. reinhardtii [62]. More recently, ATG7 phosphorylation was shown to be dependent on TOR [46], suggesting that TOR may regulate autophagy via this protein. In addition, a recent report showed that treatment of cells with concanamycin $A$, an inhibitor of the autophagic flux, repressed TAG accumulation in response to $N$ starvation. This work indicates that autophagy and vacuolar function are therefore required for lipid droplet accumulation in Chlamydomonas [63]. It is therefore likely that the activation of autophagy plays an important role in lipid droplet accumulation in response to TOR inhibition.

\section{Perspectives}

This minireview shows the importance of measuring proliferation and/or growth in connection with TAG productivity whatever the context. Manipulation of specific TOR signaling axis and the use of different ATP competitive inhibitors of TOR, at different concentrations, may help to achieve a better TAG productivity. Identifying the 
291 downstream targets of TOR needs further investigations to decipher the top secret of 292 TOR in different organisms and especially in algae that are still overlooked for TOR 293 function. More comparative studies are indeed required, particularly between different 294 algae due to their diversity and because most TOR effectors were studied in the model 295 green alga Chlamydomonas reinhardtii, which is not necessarily the best choice for 296 biotechnological applications. It is obvious that there are still many remaining questions 297 to understand the relationships between TOR and TAG accumulation in algae and 298 many studies to perform in that fascinating field. 


\section{Acknowledgments}

\section{This work was supported by the ANR SIGNAUX BioNRJ (ANR-15-CE05-0021-} 03)

\section{References}

[1] K.V. Ruggles, A. Turkish, S.L. Sturley, Making, baking, and breaking: the synthesis, storage, and hydrolysis of neutral lipids, Annual review of nutrition, 33 (2013) 413-451.

[2] Y. Li-Beisson, J.J. Thelen, E. Fedosejevs, J.L. Harwood, The lipid biochemistry of eukaryotic algae, Progress in lipid research, 74 (2019) 31-68.

[3] S.A. Shields-Menard, M. Amirsadeghi, W.T. French, R. Boopathy, A review on microbial lipids as a potential biofuel, Bioresource Technology, 259 (2018) 451-460.

[4] Y. Chisti, Biodiesel from microalgae, Biotechnology advances, 25 (2007) 294-306.

[5] T. Cavalier-Smith, Principles of protein and lipid targeting in secondary symbiogenesis: euglenoid, dinoflagellate, and sporozoan plastid origins and the eukaryote family tree, The Journal of eukaryotic microbiology, 46 (1999) 347-366.

[6] A.S. Benoiston, F.M. Ibarbalz, L. Bittner, L. Guidi, O. Jahn, S. Dutkiewicz, C. Bowler, The evolution of diatoms and their biogeochemical functions, Philosophical transactions of the Royal Society of London. Series B, Biological sciences, 372 (2017).

[7] A.E. Allen, C.L. Dupont, M. Oborník, A. Horák, A. Nunes-Nesi, J.P. McCrow, H. Zheng, D.A. Johnson, H. $\mathrm{Hu}, \mathrm{A} . \mathrm{R}$. Fernie, C. Bowler, Evolution and metabolic significance of the urea cycle in photosynthetic diatoms, Nature, 473 (2011) 203.

[8] M. Fabris, M. Matthijs, S. Carbonelle, T. Moses, J. Pollier, R. Dasseville, G.J.E. Baart, W. Vyverman, A. Goossens, Tracking the sterol biosynthesis pathway of the diatom Phaeodactylum tricornutum, New Phytologist, 204 (2014) 521-535.

[9] Q. Hu, M. Sommerfeld, E. Jarvis, M. Ghirardi, M. Posewitz, M. Seibert, A. Darzins, Microalgal triacylglycerols as feedstocks for biofuel production: perspectives and advances, The Plant journal : for cell and molecular biology, 54 (2008) 621-639.

[10] M.-H. Liang, J.-G. Jiang, Advancing oleaginous microorganisms to produce lipid via metabolic engineering technology, Progress in lipid research, 52 (2013) 395-408.

[11] Z.T. Wang, N. Ullrich, S. Joo, S. Waffenschmidt, U. Goodenough, Algal lipid bodies: stress induction, purification, and biochemical characterization in wild-type and starchless Chlamydomonas reinhardtii, Eukaryotic cell, 8 (2009) 1856-1868.

[12] R. Loewith, M.N. Hall, Target of rapamycin (TOR) in nutrient signaling and growth control, Genetics, 189 (2011) 1177-1201.

[13] A. Caron, D. Richard, M. Laplante, The Roles of mTOR Complexes in Lipid Metabolism, Annual review of nutrition, 35 (2015) 321-348.

[14] F.C. Harwood, R.I. Klein Geltink, B.P. O'Hara, M. Cardone, L. Janke, D. Finkelstein, I. Entin, L. Paul, P.J. Houghton, G.C. Grosveld, ETV7 is an essential component of a rapamycin-insensitive mTOR complex in cancer, Science advances, 4 (2018) eaar3938.

[15] D. Kazyken, Y. Kaz, V. Kiyan, A.A. Zhylkibayev, C.H. Chen, N.K. Agarwal, D. Sarbassov dos, The nuclear import of ribosomal proteins is regulated by mTOR, Oncotarget, 5 (2014) 9577-9593.

[16] Y. Luo, L. Liu, Y. Wu, K. Singh, B. Su, N. Zhang, X. Liu, Y. Shen, S. Huang, Rapamycin inhibits mSin1 phosphorylation independently of mTORC1 and mTORC2, Oncotarget, 6 (2015) 4286-4298.

[17] L.J. Smithson, D.H. Gutmann, Proteomic analysis reveals GIT1 as a novel mTOR complex component critical for mediating astrocyte survival, Genes \& development, 30 (2016) 1383-1388. 
[18] M.H. Montane, B. Menand, TOR inhibitors: from mammalian outcomes to pharmacogenetics in plants and algae, Journal of experimental botany, (2019).

[19] M. Laplante, D.M. Sabatini, mTOR signaling in growth control and disease, Cell, 149 (2012) 274293.

[20] R.A. Saxton, D.M. Sabatini, mTOR Signaling in Growth, Metabolism, and Disease, Cell, 168 (2017) 960-976.

[21] P. Crozet, L. Margalha, A. Confraria, A. Rodrigues, C. Martinho, M. Adamo, C.A. Elias, E. BaenaGonzalez, Mechanisms of regulation of SNF1/AMPK/SnRK1 protein kinases, Frontiers in plant science, 5 (2014) 190.

[22] K.M. Jamsheer, B.N. Shukla, S. Jindal, N. Gopan, C.T. Mannully, A. Laxmi, The FCS-like zinc finger scaffold of the kinase SnRK1 is formed by the coordinated actions of the FLZ domain and intrinsically disordered regions, The Journal of biological chemistry, 293 (2018) 13134-13150.

[23] P. Sanz, R. Viana, M.A. Garcia-Gimeno, AMPK in Yeast: The SNF1 (Sucrose Non-fermenting 1) Protein Kinase Complex, Experientia supplementum (2012), 107 (2016) 353-374.

[24] S.J. Ricoult, B.D. Manning, The multifaceted role of mTORC1 in the control of lipid metabolism, EMBO reports, 14 (2013) 242-251.

[25] T.I. Jeon, T.F. Osborne, SREBPs: metabolic integrators in physiology and metabolism, Trends in endocrinology and metabolism: TEM, 23 (2012) 65-72.

[26] D. Menon, D. Salloum, E. Bernfeld, E. Gorodetsky, A. Akselrod, M.A. Frias, J. Sudderth, P.H. Chen, R. DeBerardinis, D.A. Foster, Lipid sensing by mTOR complexes via de novo synthesis of phosphatidic acid, The Journal of biological chemistry, 292 (2017) 6303-6311.

[27] M.S. Yoon, Y. Sun, E. Arauz, Y. Jiang, J. Chen, Phosphatidic acid activates mammalian target of rapamycin complex 1 (mTORC1) kinase by displacing FK506 binding protein 38 (FKBP38) and exerting an allosteric effect, The Journal of biological chemistry, 286 (2011) 29568-29574.

[28] G. Lee, Y. Zheng, S. Cho, C. Jang, C. England, J.M. Dempsey, Y. Yu, X. Liu, L. He, P.M. Cavaliere, A. Chavez, E. Zhang, M. Isik, A. Couvillon, N.E. Dephoure, T.K. Blackwell, J.J. Yu, J.D. Rabinowitz, L.C. Cantley, J. Blenis, Post-transcriptional Regulation of De Novo Lipogenesis by mTORC1-S6K1-SRPK2 Signaling, Cell, 171 (2017) 1545-1558 e1518.

[29] A. Arif, F. Terenzi, A.A. Potdar, J. Jia, J. Sacks, A. China, D. Halawani, K. Vasu, X. Li, J.M. Brown, J. Chen, S.C. Kozma, G. Thomas, P.L. Fox, EPRS is a critical mTORC1-S6K1 effector that influences adiposity in mice, Nature, 542 (2017) 357-361.

[30] M.F. Wipperman, D.C. Montrose, A.M. Gotto, Jr., D.P. Hajjar, Mammalian Target of Rapamycin: A Metabolic Rheostat for Regulating Adipose Tissue Function and Cardiovascular Health, The American journal of pathology, 189 (2019) 492-501.

[31] S. D'Andrea, Lipid droplet mobilization: The different ways to loosen the purse strings, Biochimie, 120 (2016) 17-27.

[32] T.J. van Dam, F.J. Zwartkruis, J.L. Bos, B. Snel, Evolution of the TOR pathway, Journal of molecular evolution, 73 (2011) 209-220.

[33] M.H. Montané, B. Menand, ATP-competitive mTOR kinase inhibitors delay plant growth by triggering early differentiation of meristematic cells but no developmental patterning change, Journal of experimental botany, 64 (2013) 4361-4374.

[34] L. Prioretti, L. Avilan, F. Carriere, M.-H. Montane, B. Field, G. GREGORI, B. Menand, B. Gontero, The inhibition of TOR in the model diatom Phaeodactylum tricornutum promotes a get-fat growth regime, Algal Research - Biomass, Biofuels and Bioproducts, 26 (2017) 265-274.

[35] J.L. Crespo, S. Diaz-Troya, F.J. Florencio, Inhibition of target of rapamycin signaling by rapamycin in the unicellular green alga Chlamydomonas reinhardtii, Plant Physiol, 139 (2005) 1736-1749.

[36] B. Menand, T. Desnos, L. Nussaume, F. Berger, D. Bouchez, C. Meyer, C. Robaglia, Expression and disruption of the Arabidopsis TOR (target of rapamycin) gene, Proceedings of the National Academy of Sciences of the United States of America, 99 (2002) 6422-6427. 

Systemic analysis of inducible target of rapamycin mutants reveal a general metabolic switch controlling growth in Arabidopsis thaliana, The Plant journal : for cell and molecular biology, 73 (2013) 897-909. [38] D.Y. Lee, O. Fiehn, Metabolomic response of Chlamydomonas reinhardtii to the inhibition of target of rapamycin (TOR) by rapamycin, Journal of microbiology and biotechnology, 23 (2013) 923-931.

[39] S. Imamura, Y. Kawase, I. Kobayashi, T. Sone, A. Era, S.Y. Miyagishima, M. Shimojima, H. Ohta, K. Tanaka, Target of rapamycin (TOR) plays a critical role in triacylglycerol accumulation in microalgae, Plant molecular biology, 89 (2015) 309-318.

[40] J.B. Madeira, C.A. Masuda, C.M. Maya-Monteiro, G.S. Matos, M. Montero-Lomeli, B.L. BozaquelMorais, TORC1 inhibition induces lipid droplet replenishment in yeast, Molecular and cellular biology, 35 (2015) 737-746.

[41] S. Imamura, Y. Kawase, I. Kobayashi, M. Shimojima, H. Ohta, K. Tanaka, TOR (target of rapamycin) is a key regulator of triacylglycerol accumulation in microalgae, Plant signaling \& behavior, 11 (2016) e1149285.

[42] S. Mukaida, T. Ogawa, K. Ohishi, Y. Tanizawa, D. Ohta, M. Arita, The effect of rapamycin on biodiesel-producing protist Euglena gracilis, Bioscience, biotechnology, and biochemistry, 80 (2016) 1223-1229.

[43] V. Roustan, W. Weckwerth, Quantitative Phosphoproteomic and System-Level Analysis of TOR Inhibition Unravel Distinct Organellar Acclimation in Chlamydomonas reinhardtii, Frontiers in plant science, 9 (2018) 1590.

[44] S.A. Kang, M.E. Pacold, C.L. Cervantes, D. Lim, H.J. Lou, K. Ottina, N.S. Gray, B.E. Turk, M.B. Yaffe, D.M. Sabatini, mTORC1 phosphorylation sites encode their sensitivity to starvation and rapamycin, Science (New York, N.Y.), 341 (2013) 1236566.

[45] J. Juppner, U. Mubeen, A. Leisse, C. Caldana, A. Wiszniewski, D. Steinhauser, P. Giavalisco, The target of rapamycin kinase affects biomass accumulation and cell cycle progression by altering carbon/nitrogen balance in synchronized Chlamydomonas reinhardtii cells, The Plant journal : for cell and molecular biology, 93 (2018) 355-376.

[46] V. Roustan, S. Bakhtiari, P.-J. Roustan, W. Weckwerth, Quantitative in vivo phosphoproteomics reveals reversible signaling processes during nitrogen starvation and recovery in the biofuel model organism Chlamydomonas reinhardtii, Biotechnology for Biofuels, 10 (2017) 280.

[47] T. Dobrenel, E. Mancera-Martínez, C. Forzani, M. Azzopardi, M. Davanture, M. Moreau, M. Schepetilnikov, J. Chicher, O. Langella, M. Zivy, C. Robaglia, L.A. Ryabova, J. Hanson, C. Meyer, The Arabidopsis TOR Kinase Specifically Regulates the Expression of Nuclear Genes Coding for Plastidic Ribosomal Proteins and the Phosphorylation of the Cytosolic Ribosomal Protein S6, Frontiers in plant science, 7 (2016) 1611.

[48] J. Serfontein, R.E. Nisbet, C.J. Howe, P.J. de Vries, Evolution of the TSC1/TSC2-TOR signaling pathway, Science signaling, 3 (2010) ra49.

[49] E.G. Werth, E.W. McConnell, I. Couso Lianez, Z. Perrine, J.L. Crespo, J.G. Umen, L.M. Hicks, Investigating the effect of target of rapamycin kinase inhibition on the Chlamydomonas reinhardtii phosphoproteome: from known homologs to new targets, New Phytologist, 221 (2019) 247-260.

[50] M.M. Mahfouz, S. Kim, A.J. Delauney, D.P. Verma, Arabidopsis TARGET OF RAPAMYCIN interacts with RAPTOR, which regulates the activity of $\mathrm{S} 6$ kinase in response to osmotic stress signals, The Plant cell, 18 (2006) 477-490.

[51] Y. Nakamura, R. Koizumi, G. Shui, M. Shimojima, M.R. Wenk, T. Ito, H. Ohta, Arabidopsis lipins mediate eukaryotic pathway of lipid metabolism and cope critically with phosphate starvation, Proceedings of the National Academy of Sciences of the United States of America, 106 (2009) 2097820983.

[52] N. Arora, P.T. Pienkos, V. Pruthi, K.M. Poluri, M.T. Guarnieri, Leveraging algal omics to reveal potential targets for augmenting TAG accumulation, Biotechnology advances, 36 (2018) 1274-1292. 
[53] M.T. Guarnieri, A. Nag, S. Yang, P.T. Pienkos, Proteomic analysis of Chlorella vulgaris: potential targets for enhanced lipid accumulation, Journal of proteomics, 93 (2013) 245-253.

[54] M.T. Guarnieri, A. Nag, S.L. Smolinski, A. Darzins, M. Seibert, P.T. Pienkos, Examination of triacylglycerol biosynthetic pathways via de novo transcriptomic and proteomic analyses in an unsequenced microalga, PloS one, 6 (2011) e25851.

[55] J. Van Leene, C. Han, A. Gadeyne, D. Eeckhout, C. Matthijs, B. Cannoot, N. De Winne, G. Persiau, E. De Smet, G. De Jaeger, Capturing the phosphorylation and protein interaction landscape of the plant TOR kinase, Nature plants, 5 (2019) 316-327.

[56] C.P. Craddock, N. Adams, J.T. Kroon, F.M. Bryant, P.J. Hussey, S. Kurup, P.J. Eastmond, Cyclindependent kinase activity enhances phosphatidylcholine biosynthesis in Arabidopsis by repressing phosphatidic acid phosphohydrolase activity, The Plant journal : for cell and molecular biology, 89 (2017) 3-14.

[57] M. Kajikawa, Y. Sawaragi, H. Shinkawa, T. Yamano, A. Ando, M. Kato, M. Hirono, N. Sato, H. Fukuzawa, Algal dual-specificity tyrosine phosphorylation-regulated kinase, triacylglycerol accumulation regulator1, regulates accumulation of triacylglycerol in nitrogen or sulfur deficiency, Plant Physiol, 168 (2015) 752-764.

[58] M. Schulz-Raffelt, V. Chochois, P. Auroy, S. Cuine, E. Billon, D. Dauvillee, Y. Li-Beisson, G. Peltier, Hyper-accumulation of starch and oil in a Chlamydomonas mutant affected in a plant-specific DYRK kinase, Biotechnol Biofuels, 9 (2016) 55.

[59] A. Barrada, M. Djendli, T. Desnos, R. Mercier, C. Robaglia, M.H. Montane, B. Menand, A TOR-YAK1 signaling axis controls cell cycle, meristem activity and plant growth in Arabidopsis, Development, 146 (2019).

[60] I. Couso, B.S. Evans, J. Li, Y. Liu, F. Ma, S. Diamond, D.K. Allen, J.G. Umen, Synergism between Inositol Polyphosphates and TOR Kinase Signaling in Nutrient Sensing, Growth Control, and Lipid Metabolism in Chlamydomonas, The Plant cell, 28 (2016) 2026-2042.

[61] H. Shinkawa, M. Kajikawa, Y. Nomura, M. Ogura, Y. Sawaragi, T. Yamano, H. Nakagami, N. Sugiyama, Y. Ishihama, Y. Kanesaki, H. Yoshikawa, H. Fukuzawa, Algal Protein Kinase, Triacylglycerol Accumulation Regulator1, Modulates Cell Viability and Gametogenesis in Carbon/Nitrogen Imbalanced Conditions, Plant \& cell physiology, (2019).

[62] M.E. Perez-Perez, I. Couso, J.L. Crespo, The TOR Signaling Network in the Model Unicellular Green Alga Chlamydomonas reinhardtii, Biomolecules, 7 (2017).

[63] I. Couso, M.E. Perez-Perez, E. Martinez-Force, H.S. Kim, Y. He, J.G. Umen, J.L. Crespo, Autophagic flux is required for the synthesis of triacylglycerols and ribosomal protein turnover in Chlamydomonas, Journal of experimental botany, 69 (2018) 1355-1367.

\section{Figure legends}

Fig. 1. Chemical structures of the TOR inhibitors rapamycin (A) and AZD-8055 (B).

Fig. 2. Effect of TOR inhibition on lipid storage in microalgae. A) Nile Red staining of lipid droplets in Phaeodactylum tricornutum after five days of treatment with DMSO (left) or AZD-8055 $2 \mu \mathrm{M}$ (right), scale bars $10 \mu \mathrm{m}$ (modified with permission from Prioretti et al. 2017). B) BODIPY and AC-202 merged staining of lipid droplets in Chlamydomonas reinhardtii after 48h of treatment with DMSO (left) or AZD-8055 $2 \mu \mathrm{M}$ (right), scale bar $5 \mu \mathrm{m}$ (modified from Harchouni et al. 2018). C) BODIPY staining of lipid droplets in a rapamycin-sensitive strain of Cyanidioschyzon merolae expressing 
yeast FKBP12 after 48h of treatment with DMSO (left) or rapamycin $1 \mu \mathrm{M}$ (right), scale bars $10 \mu \mathrm{m}$ (modified with permission from Imamura et al. 2015). D) Nile Red staining of lipid droplets in Euglena gracilis after five days of treatment with ethanol (left) or rapamycin $1 \mu \mathrm{M}$ (right), scale bars $20 \mu \mathrm{m}$ (modified with permission from Mukaida et al. 2016).

Fig. 3. Model of effectors of TOR-dependent regulation of growth and lipid homeostasis in microalgae.

A

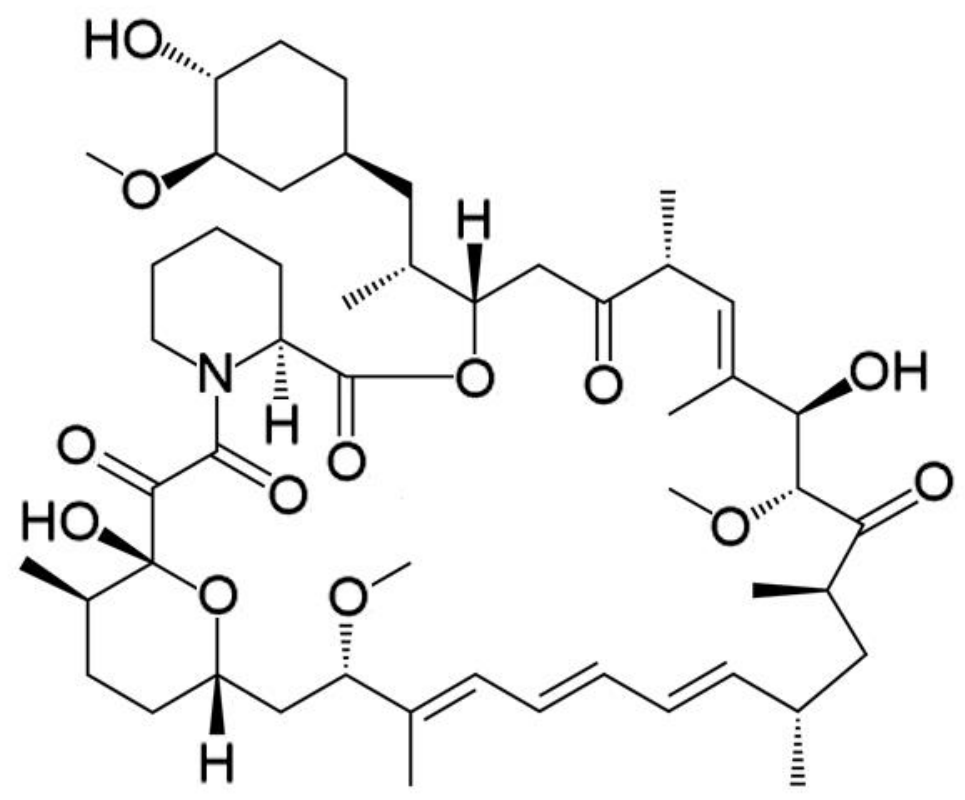

B<smiles>COc1ccc(-c2ccc3c(N4CCOC[C@H]4C)nc(N4CCOC[C@@H]4C)nc3n2)cc1CO</smiles> 

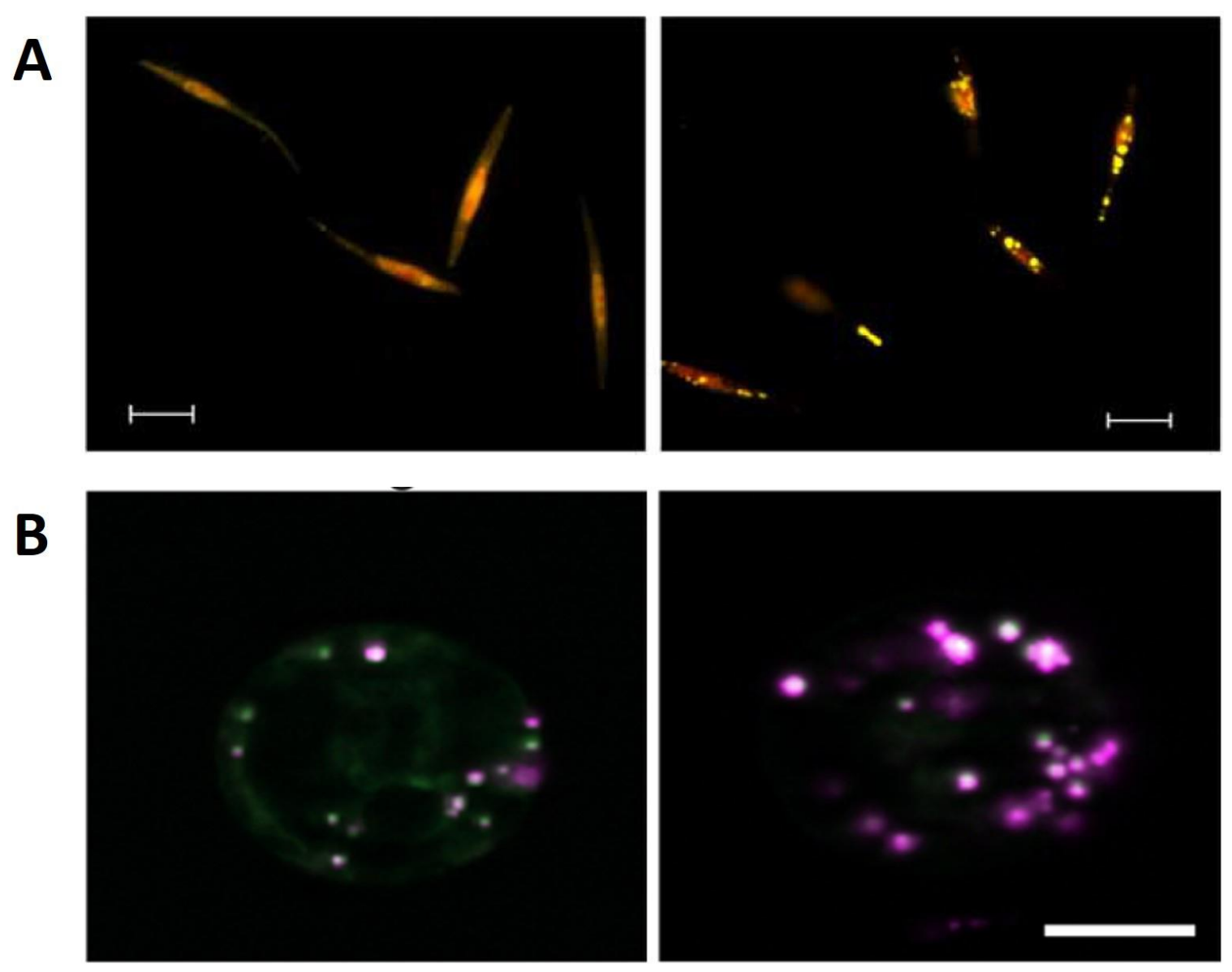

C
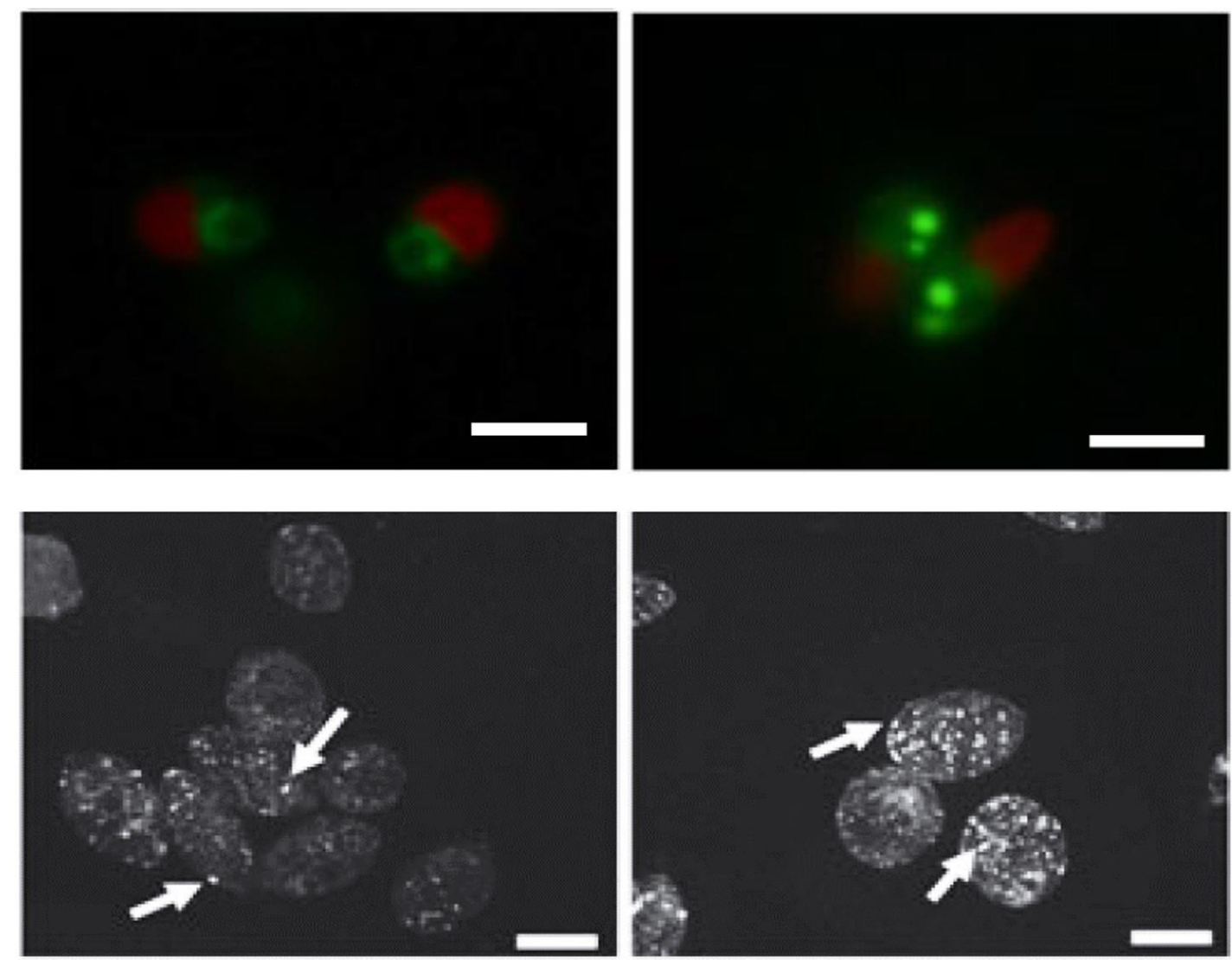


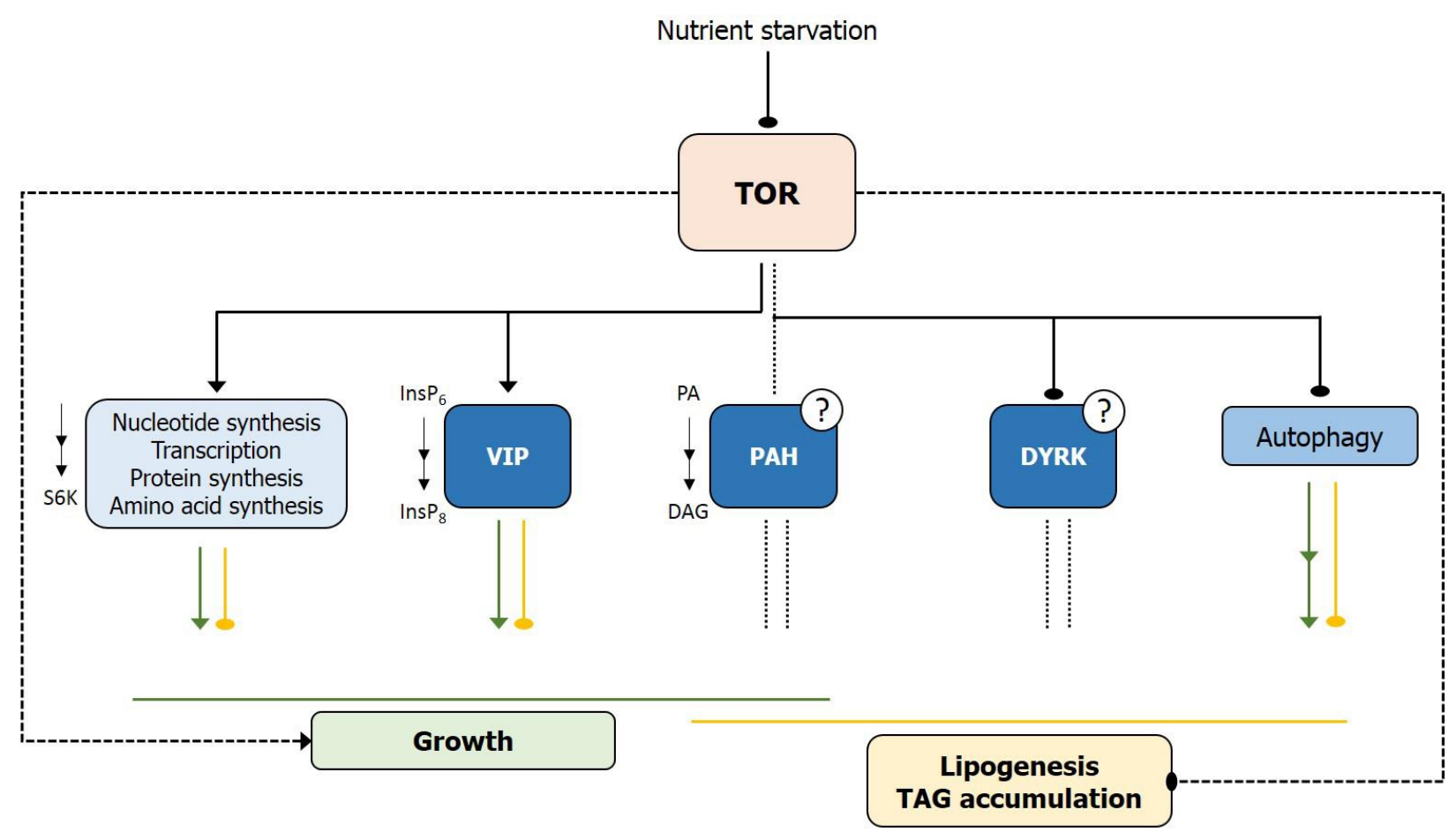

